\title{
Dynamics of microwave brightness distribution in the giant 24 August 2002 flare loop
}

\author{
Veronika E. Reznikova ${ }^{1,2}$, Victor F. Melnikov ${ }^{2}$, Kiyoto Shibasaki ${ }^{3}$, \\ Sergey P. Gorbikov ${ }^{2}$, Nikolai P. Pyatakov ${ }^{2}$, Irina N. Myagkova ${ }^{4}$, \\ Haisheng $\mathbf{J i}^{1,5}$ \\ ${ }^{1}$ Purple Mountain Observatory, Chinese Academy of Sciences, Nanjing, China \\ ${ }^{2}$ Radiophysical Research Institute (NIRFI), Nizhny Novgorod, Russia \\ ${ }^{3}$ Nobeyama Radio Observatory, NAOJ, Nagano, Japan \\ ${ }^{4}$ Lomonosov Moscow State University, Skobeltsyn Institute of Nuclear Physics, Moscow, Russia \\ ${ }^{5}$ Big Bear Solar Observatory, New Jersey Institute of Technology, Big Bear City, USA
}

\begin{abstract}
We have found a similar tendency of the spatial dynamics at $34 \mathrm{GHz}$ for all major temporal sub-peaks of the burst with the re-distribution of the brightness from the footpoints (on the rising phase of each peak) to the upper part of the loop (on the decay phase). Observed dynamics is interpreted by the re-distribution of accelerated electrons number density with their relative enhancement in the loop top. Results of diagnostics show that the ratio of non-thermal electron number density in the loop top and in the footpoint changes 7 times from the peak to decay phase. Model simulations by solving the Fokker-Planck equation allowed to determine an injection type which is able to result in necessary dynamics of energetic electrons.
\end{abstract}

Keywords. solar flare, microwave radiation, magnetic loop

\section{Observed dynamics of brightness distribution}

The total flux time profiles of the flare at $17 \mathrm{GHz}$ (thin line) and $35 \mathrm{GHz}$ (thick line) obtained with Nobeyama Polarimeter are shown in Fig. 1. Profiles have multiple emission peaks well separated from each other. Every peak is numbered at the top of Fig. 1.

Fig. 2 represents brightness evolution of $34 \mathrm{GHz}$ emission during the main temporal peak denoted as peak 1 on Fig. 1. On the rising phase of the main peak the southern footpoint (SFP) of the loop is the most bright at $34 \mathrm{GHz}$ and it remains the brightest part of the loop until the maximum. In Figs. 2a, $2 \mathrm{~b}$ we can also see two other brightness peaks: one near the opposite northern footpoint (NFP) and one near the loop top (LT), but they are much weaker. Only on the decay phase the loop top becomes relatively brighter then the footpoint sources (Fig. 2c) which almost disappear to the moment of the valley (Fig. 2d).

Interestingly, the sources of all major temporal peaks 1-6 are located at the same radio loop and the similar evolution of brightness distribution repeats itself for all these subbursts. The absolute brightness temperature of the loop top at the valley times is not reduced compared to the corresponding previous peaks of the flux time profile. Time profiles obtained for different parts of the loop showed that the emission maximum from the loop top is delayed against maxima from the footpoint sources for both frequencies. These delays are more pronounced at $34 \mathrm{GHz}$ than at $17 \mathrm{GHz}$. Furthermore, the time profile of $34 \mathrm{GHz}$ emission from the loop top is wider and its decay is slower than those from the footpoints. Time profiles of spectral index showed that almost all parts of the 

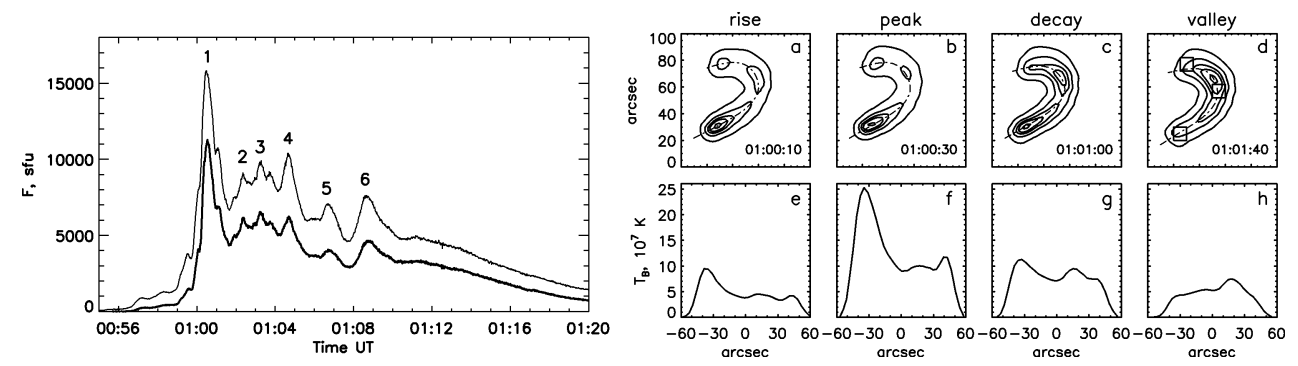

Figure 1. (left) NoRP total flux time profiles at $17 \mathrm{GHz}$ (thin line) and $35 \mathrm{GHz}$ (thick line).

Figure 2. (right) Top panel: $34 \mathrm{GHz}$ contour images of the radio source in the rise, peak and decay phases as well as at the end of the decay phase of the main peak just before the start of the second peak. Contours show 0.1, 0.4, 0.6, 0.75, 0.95 levels of the maximum brightness temperature. Dot-dashed line shows the visible flaring loop axis. Bottom panel: spatial distributions of radio brightness temperature at $34 \mathrm{GHz}$ along a visible flaring loop axes shown on the top panel at the corresponding moment of time. Abscissa is the distance along the loop, negative values correspond to southern footpoint and zero position to the loop top.

loop (with the exception of SFP) are optically thin at least at $34 \mathrm{GHz}$ since $\alpha$ is negative in the $17-34 \mathrm{GHz}$ frequency range.

\section{Discussion}

It is important that absolute brightness temperature at $34 \mathrm{GHz}$ in the loop top at the majority of valleys is not reduced compared to corresponding previous peaks of flux densities. This fact indicates the process of the accumulation of accelerated electrons in the upper part of the loop. Furthermore, the delays between microwave emissions from the loop top and footpoints as well as longer decay of the emission from the loop top are also strong evidence of the trapping and accumulation of high energy electrons in the upper part of the flaring loop (see Melnikov, Shibasaki \& Reznikova (2002)). Since this event was a limb flare with the viewing angle almost equal for all parts of the loop, the redistribution of brightness temperature reflects the re-distribution of emitting accelerated electrons along the loop.

Estimation of parameters of nonthermal electrons. To check our assumption we estimated ratios of electron number densities in the loop top and southern footpoint $N_{L T} / N_{S F P}$ for different time moments. The estimations obtained by fitting observed fluxes at $17 \mathrm{GHz}$ and $34 \mathrm{GHz}$ by the spectrum for both sources using exact formulas given by Ramaty (1969), Fleishman \& Melnikov (2003) for GS emissivity and absorption coefficient. Electron energy spectral index $\delta$ was derived from HXR data obtained by detector SONG aboard of space solar observatory CORONAS-F under the assumption of thick target model. At the maximum of peak $1 \delta_{H X}=2.6$, at the valley 1 time $\delta_{H X}=3.1$ for channels $53-150 \mathrm{keV}$ and $150-500 \mathrm{keV}$. All other parameters were taken from the observations: an ambient plasma density $8 \times 10^{10} \mathrm{~cm}^{-3}$ (from GOES/SXT at peak 1); the source depth $3.6 \times 10^{8} \mathrm{~cm}$; the viewing angle $84^{\circ}$.

Estimation showed the ratio $N_{L T} / N_{S F P}$ increases about 7 times from peak 1 time to the end of decay (valley 1) for the mirror ratio 5 . Diagnostics of plasma parameters in the loop gave magnetic field strength in the $\mathrm{SFP}$ source $B_{S F P} \approx 1000 \mathrm{G}$, and in the loop top $B_{L T} \approx 200 \mathrm{G}$; number densities of accelerated electrons with energies more than 500 $\mathrm{keV}$ in SFP source $N_{S F P}=3 \times 10^{4} \mathrm{~cm}^{-3}$ and $N_{L T}=9 \times 10^{5} \mathrm{~cm}^{-3}$ at the maximum of peak 1 and $N_{S F P}=3 \times 10^{3} \mathrm{~cm}^{-3}, N_{L T}=6 \times 10^{5} \mathrm{~cm}^{-3}$ at the valley time. Thus, 


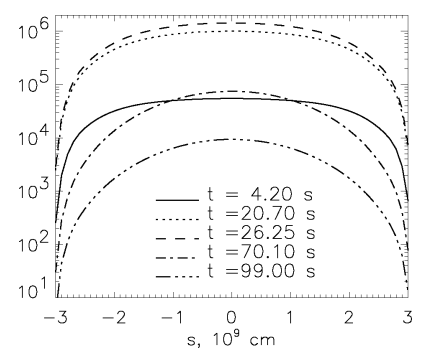

Figure 3. Results of the model simulations using the Fokker-Planck equation. The electron distribution functions $f(E, \mu, s, t)$ for the rising phase of injection are shown by solid and dotted lines, near the injection maximum by dashed line, and for the decay phase by dot-dashed and dot-dot-dot-dashed lines.

diagnostics showed that already at peak 1 time number density in LT is about 30 times higher than in the SFP.

Model calculations using Fokker-Planck equation. On Fig. 3 we present the result of calculation of the time evolution of energetic electron number density along the loop. It is shown for electrons with pitch-angle $85^{\circ}$ and energy $E=405 \mathrm{keV}$ only for the case when injection is isotropic and continuous along the loop. Magnetic mirror ratio $m=5$. The flat distribution function at the rising phase should give a radio brightness peak in the footpoint with the strongest magnetic field. Our diagnostics shows that even at the peak time accelerated electron number density is higher in the loop top than in the southern foot point. We can see the similar result in this model, where $N_{L T} / N_{F P}=7 \div 100$ at the peak time, considering the location of footpoint $s>2.4 \times 10^{9} \mathrm{~cm}$. At the decay phase the density in the central part of the trap becomes well pronounced. Such dynamics of the particle spatial distribution are caused by pitch-angle scattering due to Coulomb collisions which leads to preferable accumulation of energetic electrons near the center of the trap.

\section{Conclusions}

For the first time we have found the cyclical dynamics in radio brightness distribution along the loop, synchronized with major temporal peaks: re-distribution of the radio brightness from the footpoints (on the rising phase) to the upper part of the loop (on the decay phase). Since this is the limb event with the loop plane is almost perpendicular to the line of sight, the re-distribution of $T_{B}$ directly reflects the re-distribution of accelerated electrons number density with they relative enhancement in the loop top. As a result of our diagnostics we have obtained that the ratio $N_{L T} / N_{S F P}$ increases by about 7 times from peak 1 to the end of decay (valley 1) and already at peak 1 the electron number density in the LT is higher than in the SFP. This result of diagnostics is in a good agreement with our calculation of the time evolution of energetic electron number density for the model with isotropic injection which occurs continuous along the loop.

The work was partly supported by RFBR grants No. 06-02-39029, 06-02-16295, 07-0201066, NSFC grant 10473024 CNSF 10833007, the 973 project with No. 2006CB806302, and Bairen project.

\section{References}

Fleishman, G. D., \& Melnikov, V. F. 2003, ApJ, 584, 1071

Melnikov, V. F., Shibasaki, K., \& Reznikova., V. E. 2002, ApJ, 480, L185

Ramaty, R. 1969, ApJ, 158, 753 